\title{
Selective BTEX Sensing with Laser Absorption and DNNs
}

\author{
Mhanna Mhanna, Mohamed Sy, Aamir Farooq
}

King Abdullah University of Science and Technology (KAUST), Physical Science and Engineering Division, Thuwal, 23955-6900, Saudi Arabia

Author e-mail address: aamir.farooq@ kaust.edu.sa

\begin{abstract}
Selective and simultaneous BTEX measurements are performed utilizing a $3.3 \mu \mathrm{m}$ DFBICL. The sensor is based on wavelength tuning and deep neural networks (DNNs) to distinguish the broadband absorbance spectra of BTEX species. (C) 2021 The Author(s)
\end{abstract}

\section{Introduction}

Human activity has deleterious impacts on air quality, in large part due to transportation and industrial emissions. Petrochemical industries are the major emitters of volatile organic compounds (VOCs). In particular, benzene, toluene, ethylbenzene and xylene isomers (BTEX) are emitted from engine exhaust, gasoline service stations, refineries, painting and rubber industries [1]. The ubiquity and adverse effects of these species, even at the ppm level, on human health have raised tremendous concerns in recent years. Short-term exposure to benzene, for example, can lead to acute effects like dizziness or drowsiness, while long-term exposure can cause cancer [2]. Inhaling toluene can cause severe damages to the brain, liver and kidneys [3]. Exposure to ethylbenzene irritates the respiratory tract and eyes [4]. Distortion in the nervous system and lack of muscle coordination have been reported due to xylene isomers [5]. It is, therefore, extremely important to have reliable, accurate and portable sensors for these harmful pollutants. The similar molecular structure of BTEX species cause the existing sensors to have poor selectivity. This work reports the development of a selective BTEX sensor based on a mid-IR laser source and deep neural networks (DNNs) to differentiate between the similar absorbance spectra of BTEX molecules.

\section{Sensor Description}

Based on the IR spectra of BTEX in the PNNL database [6], the best wavelength to measure these is near $14 \mu \mathrm{m}$. However, due to the unavailability of commercial laser sources in that region, we selected the C-H stretching bands near $3.3 \mu \mathrm{m}$. The wavelength region near $3040 \mathrm{~cm}^{-1}$ has been chosen based on detailed interference analysis in a previous work [7]. Figure 1 shows the simulated absorbance spectra of BTEX species $\left(\mathrm{T}=25^{\circ} \mathrm{C}, \mathrm{P}=1 \mathrm{~atm}, \mathrm{~L}=30\right.$ $\mathrm{cm}, \chi=1000 \mathrm{ppm}$ ) [6] which exhibit similar structure due to the $\mathrm{C}-\mathrm{H}$ stretching in the aromatic rings of these species. This makes it highly challenging to measure BTEX species selectively. Here, wavelength-scanning and deep neural networks (DNNs) were employed to overcome this difficulty. The laser source is a DFB interband cascade laser (DFBICL, Nanoplus) that emits $\sim 1-2 \mathrm{~mW}$ near $3.3 \mu \mathrm{m}$. The laser is transmitted through a $30 \mathrm{~cm}$ sampling cell with two ZnSe windows (Thorlabs) and it is collected on a DC-coupled, TE-cooled photodetector (bandwidth of $1.5 \mathrm{MHz}$, Vigo Systems). Figure 2 shows the sensor setup.

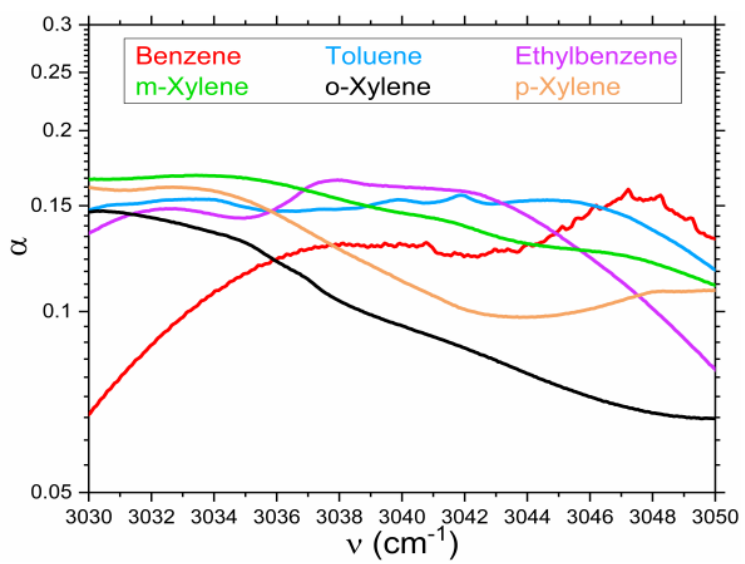

Fig. 1. BTEX absorbance spectra at $\mathrm{T}=25^{\circ} \mathrm{C}, \mathrm{P}=1 \mathrm{~atm}, \mathrm{~L}=30 \mathrm{~cm}$, $\chi=1000 \mathrm{ppm}$ over $3030-3050 \mathrm{~cm}^{-1}[6]$.

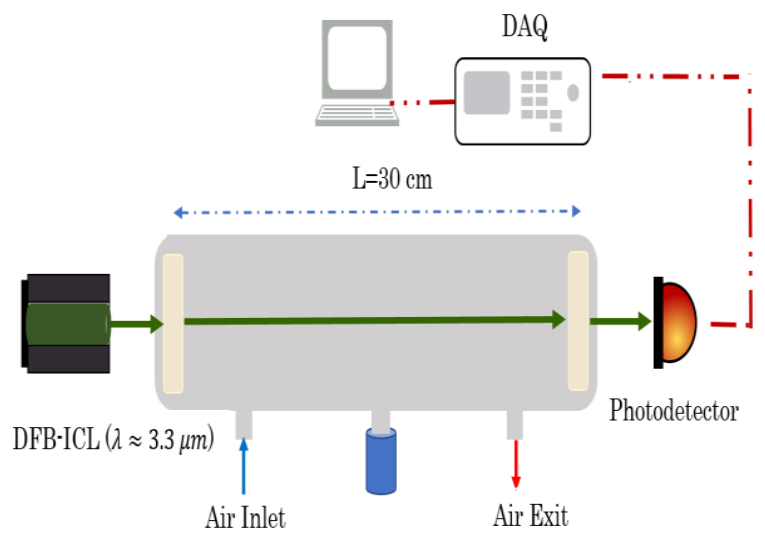

Fig. 2. Optical schematic of the BTEX sensor.

\section{Results and Discussion}

The similarly-shaped broad BTEX spectra impose severe challenges on traditional fitting algorithms. Multidimensional linear regression (MLR) was used previously to distinguish spectra of benzene, ethylene and methane [8]. However, predictive ability of MLR is dramatically compromised in the case of BTEX species. Hence, various 
machine learning algorithms like support vector machines (SVM), gradient boosting (XGB) and deep neural networks (DNNs) were explored here. To test the ability of this sensor for selective and simultaneous BTEX measurements, experiments were carried out with various mixtures prepared in the lab. The 10-fold cross-validation results for 5400 simulated and 132 measured absorbance spectra are shown in Table 1. The best performance was observed with DNN comprising of 3 hidden layers of 64, 32 and 16 nodes, respectively. Thereafter, DNN model was trained and tested based on a random 80/20 split. Figure 3 shows the results of applying DNN on gas mixtures containing various BTEX concentrations, where each measurement was repeated 5 times. The good agreement between the measured and manometric concentrations, along with the good measurement repeatability, validates the capability of this diagnostic for selective and simultaneous BTEX measurements.

Table 1: 10-fold cross validation results of MLR, SVM, XGB and DNN.

\begin{tabular}{ccccc}
\hline Fitting parameter & MLR & SVM & XGB & DNN \\
\hline RMSE & 0.096 & 0.093 & 0.041 & 0.024 \\
$\mathrm{R}^{2}$ & 0.722 & 0.774 & 0.945 & 0.985 \\
\hline
\end{tabular}

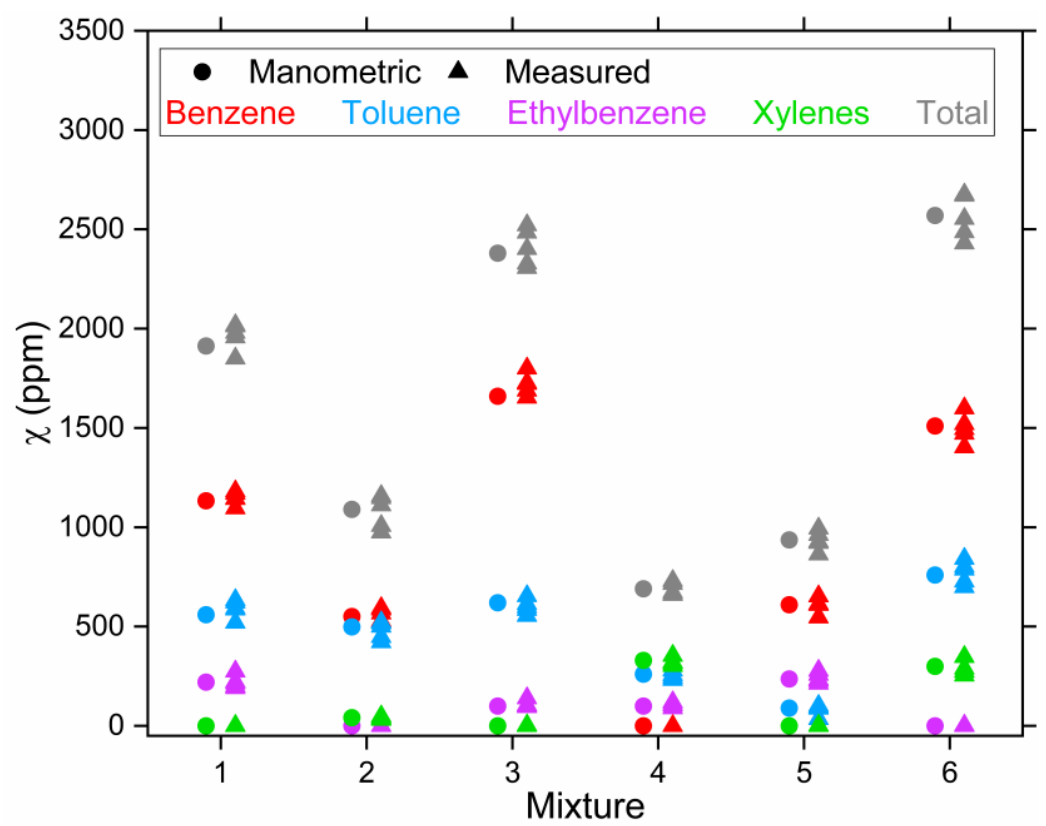

Figure 3: Comparison of predicted BTEX concentrations with manometric values.

\section{Conclusions}

Selective and simultaneous BTEX measurements are performed with an absorption-based laser sensor. The sensor was validated with real gas samples where best prediction results were obtained with the help of a DNN algorithm. Major health risks can be avoided with this sensor in petrochemical, painting and rubber industries where BTEX emissions are likely.

\section{References}

[1] Zhang, Yujie, et al. "Levels, sources and health risks of carbonyls and BTEX in the ambient air of Beijing, China." Journal of Environmental Sciences 24.1 (2012): 124-130.

[2] Wilbur, S., et al. "ATSDR evaluation of health effects of benzene and relevance to public health." Toxicology and industrial health 24.5-6 (2008): 263-398

[3] Dorsey, Alfred. "Toxicological profile for toluene." (2000).

[4] Taylor, Jessilynn. Toxicological Profile for Ethylbenzene. US Department of Health \& Human Services, Public Health Service, Agency for Toxic Substances and Disease Registry, 2010.

[5] Fay, Mike, J. Risher, and Jewell D. Wilson. "Toxicological profile for xylene." (2007).

[6] Sharpe, Steven W., et al. "Gas-phase databases for quantitative infrared spectroscopy." Applied spectroscopy 58.12 (2004): 1452-1461.

[7] Mhanna, Mhanna, et al. "Cavity-Enhanced Measurements of Benzene for Environmental Monitoring." IEEE Sensors Journal (2020).

[8] Mhanna, Mhanna, et al. "A Cavity-Enhanced Sensor for Benzene Detection." Optical Sensors. Optical Society of America, 2020. 